\title{
ON THE HOMOLOGY OF BRANCHED COVERINGS OF 3-MANIFOLDS
}

\author{
JUN UEKI
}

\begin{abstract}
Following the analogies between 3-manifolds and number rings in arithmetic topology, we study the homology of branched covers of 3-manifolds. In particular, we show some analogues of Iwasawa's theorems on ideal class groups and unit groups, Hilbert's Satz 90, and some genus-theory-type results in the context of 3-dimensional topology. We also prove that the 2-cycles valued Tate cohomology of branched Galois covers is a topological invariant, and we give a new insight into the analogy between 2-cycle groups and unit groups.
\end{abstract}

\section{$\S 1$. Introduction}

The analogy between 3-dimensional topology and number theory was first pointed out by Mazur [Ma] in the 1960s, and it has been studied systematically by Kapranov, Reznikov [Re], and Morishita [Mo3], [Mo4]. In their analogies, for example, knots and 3-manifolds correspond to primes and number rings, respectively. The study of these analogies is now called arithmetic topology.

The purpose of this article is to study the homology of branched coverings of 3-manifolds by following the analogies in arithmetic topology. In particular, we show the topological analogues of Iwasawa's theorems on ideal class groups and unit groups in number field extensions, and we give some applications to topological analogues of genus theory. In the course of our proof, we show the 3-manifold analogue of Hilbert's Satz 90. In addition, we prove that the 2-cycles valued Tate cohomology of branched covers is a topological invariant, which gives a new insight into the analogy between 2-cycles and units.

Received December 5, 2011. Revised October 26, 2012. Accepted March 5, 2013.

First published online November 26, 2013.

2010 Mathematics Subject Classification. Primary 57M12; Secondary 12G05, 52M27, 11R29, 11R32.

(C) 2014 by The Editorial Board of the Nagoya Mathematical Journal 
This article is organized in the following manner. In Section 2, we review the basic analogies between 3-dimensional topology and number theory, which will be used throughout this paper, and Hilbert theory for 3-manifold following [Mo4]. In Section 3, we recall Iwasawa's theorems and so forth on ideal class groups and unit groups in number field extensions, and we state their topological analogues as our main theorems. We also give some remarks on the 2-cycles valued Tate cohomology of branched covers. In Section 4, we prove our main theorems. In Section 5, as applications of our theorems, we give genus theory-type results, which give balances between homology groups and branch information.

\section{§2. Hilbert theory for 3-manifolds}

Hilbert theory deals with, in a group-theoretic manner, the decomposition of a prime in a finite Galois extension of number fields. In this section, we recollect Hilbert theory for 3-manifolds, which describes the decomposition of a knot in a finite Galois branched cover of 3-manifolds.

First, we recall in the following table some of the basic analogies between number theory and 3-dimensional topology which will be used in this paper. For a number field $k, \mathcal{O}_{k}$ denotes the ring of integers in $k$.

\begin{tabular}{|c|c|}
\hline Number ring $\operatorname{Spec}\left(\mathcal{O}_{k}\right)$ & 3-dimensional manifold $M$ \\
\hline Prime ideal $\mathfrak{p}$ & Knot $K$ \\
Prime ideals $S=\left\{\mathfrak{p}_{1}, \ldots, \mathfrak{p}_{r}\right\}$ & Link $L=\left\{K_{1}, \ldots, K_{r}\right\}$ \\
\hline $\begin{array}{c}\text { Étale fundamental group } \pi_{1}\left(\operatorname{Spec}\left(\mathcal{O}_{k}\right)\right) \\
\pi_{1}\left(\operatorname{Spec}\left(\mathcal{O}_{k}\right)-S\right)\end{array}$ & $\pi_{1}(M)$ \\
\hline Number field extension $\ell / k$ & Link group $\pi_{1}(M-L)$ \\
\hline Ideal group $I(k)$ & Branched) Cover $h: N \rightarrow M$ \\
$k^{*} \rightarrow I(k) ; a \mapsto(a)$ & $C_{2}(M, \mathbb{Z}) \rightarrow Z_{1}(M, \mathbb{Z}) ; S \mapsto \partial S$ \\
\hline Principal ideal group $P(k)$ & 1 -boundary group $B_{1}(M, \mathbb{Z})$ \\
\hline Ideal class group $C l(k)=I(k) / P(k)$ & First homology \\
& $H_{1}(M, \mathbb{Z})=Z_{1}(M, \mathbb{Z}) / B_{1}(M, \mathbb{Z})$ \\
\hline Unit group $\mathcal{O}_{k}^{*}$ & Second homology \\
& $H_{2}(M, \mathbb{Z})$, or 2 -cycles $Z_{2}(M, \mathbb{Z})$ \\
\hline Artin reciprocity & Hurewicz isomorphism \\
$C l(k) \cong$ Gal $\left(k_{a b}^{u r} / k\right) \cong \pi_{1}\left(\operatorname{Spec}\left(\mathcal{O}_{k}\right)\right)^{a b}$ & $H_{1}(M, \mathbb{Z}) \cong$ Gal $\left(M_{a b} / M\right) \cong \pi_{1}(M)^{a b}$ \\
$k_{a b}^{u r}:$ Hilbert class field of $k$ & $M_{a b}:$ maximal abelian cover of $M$ \\
\hline
\end{tabular}


Particularly, we have parallel exact sequences

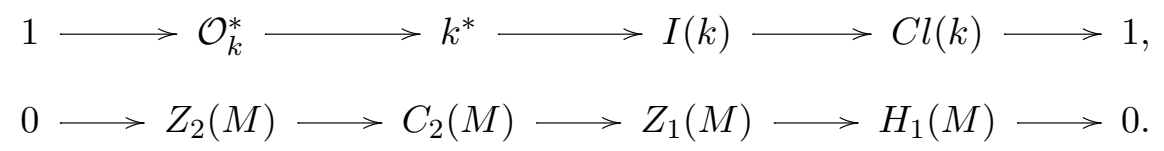

Because of this, in this paper we consider 2-cycles of 3-manifolds, rather than 2-homologies, as an analogue of units of $\mathcal{O}_{k}$, and we pursue some analogies with number fields (see Theorems 5, 10, and 11 below). Reznikov [Re] also considered surfaces without boundaries for this.

Since $C l(k)$ is finite, to get the precise analogies for manifold it is natural to assume that $H_{1}(M, \mathbb{Z})$ is finite, that is, that manifolds are rational homology 3 -spheres. However, by considering the torsion subgroup $\operatorname{tor}\left(H_{1}(M, \mathbb{Z})\right)$ as the counterpart of $C l(k)$, some analogies work (see [Si], [Mn1]).

For more analogies, we refer to [Mo3], [Mo4], or [Mn2].

Now, based on the above dictionary, we present a topological analogue of Hilbert theory following [Mo4, Chapter 3].

Let $h: N \rightarrow M$ be a finite Galois covering of connected oriented closed 3manifold branched over a link $L \subset M$, let $X:=M-L$, let $Y:=N-h^{-1}(L)$, let $G:=\operatorname{Gal}(Y / X)=\operatorname{Gal}(N / M)$, and let $n:=\# G(1)$. Let $K$ be a knot in $M$ which is a component of $L$ or disjoint from $L$, and suppose that $h^{-1}(K)=$ $K_{1} \cup \cdots \cup K_{r}\left(r=r_{K}\right.$-component link). For a tubular neighborhood $V_{K}$ of $K$, let $V_{K_{i}}$ be the connected component of $h^{-1}\left(V_{K}\right)$ containing $K_{i}$. (They are canonical up to isotopy, in any category.) Note that $G$ acts transitively on the set of knots $S_{K}:=\left\{K_{1}, \ldots, K_{r}\right\}$ lying over $K$. We call the stabilizer $D_{K_{i}}$ of $K_{i}$ the decomposition group of $K_{i}$ :

$$
D_{K_{i}}:=\left\{g \in G \mid g\left(K_{i}\right)=K_{i}\right\} .
$$

Since we have the bijection $G / D_{K_{i}} \cong S_{K}$ for each $i$, \# $D_{K_{i}}=n / r$ is independent of $K_{i}$. In fact, if $g\left(K_{i}\right)=K_{j}$, then $D_{K_{j}}=g D_{K_{i}} g^{-1}$. Since each $g \in G$ induces a homeomorphism $\left.g\right|_{\partial V_{K_{i}}}: \partial V_{K_{i}} \cong \partial V_{g\left(K_{i}\right)},\left.g\right|_{\partial V_{K_{i}}}$ is a covering transformation of $\partial V_{K_{i}}$ over $\partial V_{K}$ for each $g \in D_{K_{i}}$, and the corresponding $\left.g \rightarrow g\right|_{\partial V_{K_{i}}}$ gives an isomorphism

$$
D_{K_{i}} \cong \operatorname{Gal}\left(\partial V_{K_{i}} / \partial V_{K}\right)
$$

The Fox completion of the subcovering space of $Y$ over $X$ corresponding to $D_{K_{i}}$ is called the decomposition covering space of $K_{i}$ and is denoted by 
$Z_{K_{i}}$. The map $g \mapsto \bar{g}=\left.g\right|_{\partial V_{K_{i}}}$ induces the homomorphism

$$
D_{K_{i}} \rightarrow \operatorname{Gal}\left(K_{i} / K\right)
$$

whose kernel is called the inertia group of $K_{i}$ and is denoted by $I_{K_{i}}$ :

$$
I_{K_{i}}:=\left\{g \in D_{K_{i}} \mid \bar{g}=\operatorname{id}_{K_{i}}\right\} .
$$

If $K_{j}=g\left(K_{i}\right)(g \in G)$, one has $I_{K_{j}}=g I_{K_{i}} g^{-1}$, and hence \# $I_{K_{i}}$ is independent of $K_{i}$. Set $e=e_{K}:=\# I_{K_{i}}$. The Fox completion of the subcovering space of $Y$ over $X$ corresponding to $I_{K_{i}}$ is called the inertia covering space of $K_{i}$ and is denoted by $T_{K_{i}}$ :

$$
\begin{aligned}
& N \longrightarrow T_{K_{i}} \longrightarrow Z_{K_{i}} \longrightarrow M \\
& 1 \stackrel{e}{\longrightarrow} I_{K_{i}} \stackrel{f}{\longrightarrow} D_{K_{i}} \stackrel{r}{\longrightarrow} G
\end{aligned}
$$

Here we have the equalities

$$
\# D_{K_{i}}=e f, \quad \# I_{K_{i}}=e, \quad \# \operatorname{Gal}\left(K_{i} / K\right)=: f .
$$

By comparing the orders, we see that the homomorphism $D_{K_{i}} \ni g \mapsto \bar{g} \in$ $\operatorname{Gal}\left(K_{i} / K\right)$ is surjective:

$$
1 \longrightarrow I_{K_{i}} \longrightarrow D_{K_{i}} \longrightarrow \operatorname{Gal}\left(K_{i} / K\right) \longrightarrow 1 \quad \text { (exact). }
$$

Let $K_{i, T}$ be the image of $K_{i}$ under $N \rightarrow T_{K_{i}}$, and let $K_{i, Z}$ be the image of $K_{i, T}$ under $T_{K_{i}} \rightarrow Z_{K_{i}}$. Then one has the following.

Theorem ([Mo4, Chapter 3]). The map $N \rightarrow T_{K_{i}}$ is a branched cover of degree $e$ such that the branching index of $K_{i}$ over $K_{i, T}$ is e. The map $T_{K_{i}} \rightarrow Z_{K i}$ is a cyclic cover of degree $f$ such that the covering degree of $K_{i, T}$ over $K_{i, Z}$ is $f$. The map $Z_{K_{i}} \rightarrow M$ is a cover of degree $r$ such that $K$ is completely decomposed into an $r$-component link containing $K_{i, Z}$ as a component.

\section{$\S 3$. Iwasawa's theorems and their topological analogues}

\subsection{Iwasawa's theorems}

Now we recall Iwasawa's theorems on ideal class groups in number field extensions. 
TheOREM 1 ([I2]). Let $\ell / k$ be a finite extension of number fields (finite extensions of $\mathbb{Q}$ ), and suppose that $\ell / k$ has no nontrivial unramified abelian subextension; that is, if $\ell / m / k$ and $m / k$ are unramified abelian, then $m=k$. (This assumption is obviously satisfied if $\ell / k$ is totally ramified at some prime p.) Then the norm map of ideal class groups $N_{\ell / k}: C l(\ell) \rightarrow C l(k)$ is surjective, and hence $\# \mathrm{Cl}(k) \mid \# C l(\ell)$.

Theorem 2 ([I2], [W, Theorem 10.4]). Let $\ell / k$ be a Galois extension of number field with degree $p^{\nu}$, where $p$ is a prime number and $\nu$ is a natural number, and suppose that $\ell / k$ is ramified over at most one prime. Then $p \mid \# C l(\ell)$ implies that $p \mid \# C l(k)$. In particular, when $k=\mathbb{Q}$, it follows that $p X \# C l(\ell)$.

Corollary $3([\mathrm{~F}])$. We have

$$
p\left|\# C l\left(\mathbb{Q}\left(\zeta_{p}\right)\right) \Longleftrightarrow p\right| \# C l\left(\mathbb{Q}\left(\zeta_{p^{\nu}}\right)\right),
$$

where $p$ is a prime number, $\nu$ is a natural number, and $\zeta_{n}$ is a primitive nth root of unity.

Remark. For the asymptotic behavior of the order of the p-part of $C l\left(\mathbb{Q}\left(\zeta_{p^{\nu}}\right)\right)$ as $\nu \rightarrow \infty$, Iwasawa's class number formula [I1, Theorem 12] is known.

We also recall Iwasawa's theorems on unit groups in number field extensions. We denote by $\widehat{H}^{n}(G, A)$ the Tate cohomology of a group $G$ acting on an abelian group $A$, which is equal to the Galois cohomology $H^{n}(G, A)$ if $n>0$ (see [Se, Chapter 8]). The following lemma proves Theorem 5 .

Lemma 4 (Hilbert's Satz 90). Let $\ell / k$ be a finite Galois extension of number fields with Galois group G; then we have

$$
\widehat{H}^{1}\left(G, \ell^{*}\right)=0 .
$$

Theorem 5 ([I1, Chapter 2]). Let $\ell / k$ be a finite Galois extension of number fields with Galois group $G$; then

$$
\widehat{H}^{1}\left(G, \mathcal{O}_{k}^{*}\right) \cong P(\ell)^{G} / P(k),
$$

where $P(k)$ is the group of principal ideals in $k$ and $P(\ell)^{G}$ is the group of principal ideals in $k$ on which $G$ acts trivially. 


\subsection{Main theorems}

Next, we state our main theorems, which are topological analogues of Iwasawa's theorems and so forth given in Section 3.1. Hereafter, all manifolds in the statements are assumed to be closed (i.e., compact and $\partial M=\phi$ ), oriented, and connected. Note that a finite branched cover over a closed manifold is always closed.

On the first homologies, we have Theorems 6 and 7 and Corollary 8, which are regarded as analogues of Theorems 1 and 2 and Corollary 3.

THEOREM 6. Let $h: N \rightarrow M$ be a (branched) cover of $n$-manifolds, and suppose that $h$ has no nontrivial unbranched abelian subcover; that is, if $h=g \circ f: N \rightarrow S \rightarrow M$ for some continuous map $f$ and some unbranched abelian cover $g$ of $M$, then $g$ is a homeomorphism. (This assumption is satisfied if $n=3$ and $h$ is totally branched over some knot $K$.) Then the induced $\operatorname{map}_{*}: H_{1}(N, \mathbb{Z}) \rightarrow H_{1}(M, \mathbb{Z})$ is surjective. In particular, if $N$ and $M$ are rational homology spheres, then $\# H_{1}(M) \mid \# H_{1}(N)$.

THEOREM 7. Let $h: N \rightarrow M$ be a (branched) Galois cover of 3-manifolds branched over at most one knot and of degree $p^{\nu}$, where $p$ is a prime number and $\nu$ is a natural number. If $N$ and $M$ are rational homology spheres, then $p \mid \# H_{1}(N, \mathbb{Z})$ implies that $p \mid \# H_{1}(M, \mathbb{Z})$. In particular, when $M$ is $S^{3}$ or an integral homology sphere, it follows that $p \nmid \# H_{1}(N, \mathbb{Z})$.

COROllary 8. Let $M_{n}$ denote the cyclic branched cover of $S^{3}$ branched over a knot $K$, and of positive degree $n$. Assume that $M_{n}$ are all rational homology spheres. Let $p$ be a prime number, let $m$ be a positive integer, and let $\nu$ be a natural number. Then we can show that

$$
p\left|\# H_{1}\left(M_{m}, \mathbb{Z}\right) \Longleftrightarrow p\right| \# H_{1}\left(M_{m p^{\nu}}, \mathbb{Z}\right) .
$$

REMARK. For the asymptotic behavior of the order of $p$-part of $H_{1}\left(M_{m p^{\nu}}\right.$, $\mathbb{Z})$, we refer to $[\mathrm{HMM}$, Chapter 5$]$ and $[\mathrm{KM}]$.

On 2-chains and 2-cycles valued Tate cohomology groups of Galois covers, we have Lemma 9 and Theorem 10, which are 3-manifold analogues of Lemma 4 and Theorem 5.

For the remainder of this article, we assume that manifolds admit finite $\mathrm{CW}$-structures that are compatible to covering maps and include branching 
sets, and we fix such structures on them. ${ }^{\dagger}$ Then we can consider branched Galois covers with Galois group $G$ as $G$-complexes (see [B, Chapter I.4]). The following Lemma 9 implies our Theorem 10.

Lemma 9 (Analogue of Hilbert's Satz 90). Let $h: N \rightarrow M$ be a Galois cover of 3-manifolds branched over some link with Galois group $G$; then

$$
\widehat{H}^{1}\left(G, C_{2}(N, \mathbb{Z})\right)=0 .
$$

TheOREM 10. Let $h: N \rightarrow M$ be a Galois cover of 3-manifolds branched over some link; then

$$
\widehat{H}^{1}\left(G, Z_{2}(N, \mathbb{Z})\right) \cong B_{1}(N, \mathbb{Z})^{G} / h^{!} B_{1}(M, \mathbb{Z})
$$

where $B_{1}(N, \mathbb{Z})^{G}$ is the $G$-invariant subgroup of 1-boundaries.

Here, we denote by $h^{!}: C_{*}(M, \mathbb{Z}) \hookrightarrow C_{*}(N, \mathbb{Z})$ the canonical injection, called transfer, which is defined as follows. For any open chain $c \subset M$, take one connected component of $h^{-1}(c)$, say, $c_{1}$, and put $h^{!}(c)=\sum_{\sigma \in G} \sigma c_{1}$ and extend linearly on the whole $C_{*}(M, \mathbb{Z})$.

Although $Z_{2}(N, \mathbb{Z})$ depends on the choice of $\mathrm{CW}$-structure, there is a remarkable fact.

THEOREM 11. We have the following isomorphism:

$$
\widehat{H}^{r}\left(G, Z_{2}(N, \mathbb{Z})\right) \stackrel{\cong}{\rightrightarrows} \widehat{H}^{r}\left(G, Z_{2}^{\text {sing }}(N) / Z_{2}^{\text {sing }}\left(h^{-1}(L)\right)\right),
$$

where $Z_{2}^{\text {sing }}(N)$ is the singular 2-cycle group of $N$ and $L$ is the branching link of $h$. Especially, the Tate cohomology $\widehat{H}^{r}\left(G, Z_{2}(N, \mathbb{Z})\right)(r \in \mathbb{Z})$ is a topological invariant of branched covers, that is, independent of the choice of $C W$-structure.

\section{REMARKS.}

(1) Theorems 10 and 11 strengthen our reason to consider the 2-cycle group $Z_{2}(N, \mathbb{Z})$, rather than the 2-homology group $H_{2}(N, \mathbb{Z})$, as an analogue of unit group $\mathcal{O}_{k}^{*}$. There are two merits of our point of view.

\footnotetext{
${ }^{\dagger}$ It is known that compact manifold $M$ always admits CW-structure if $\operatorname{dim} M \neq 4$, while the case for $\operatorname{dim} M=4$ is an open problem (see [H, p. 529]). Although a CWstructure on a 3-manifold is not unique, we can discuss closer analogies with number fields, such as Lemma 9 and Theorems 10 and 11 below, once such a structure is chosen.
} 
(i) $Z_{2}(N, \mathbb{Z})$ has more information (of the topology of $h: N \rightarrow M$ ) than does $H_{2}(N, \mathbb{Z})$. Indeed, if $N$ is a rational homology sphere, $H_{2}(N, \mathbb{Z})$ is always finite, and $\widehat{H}^{q}\left(G, H_{2}(N, \mathbb{Z})\right)=0$, while usually $\widehat{H}^{q}\left(G, Z_{2}(N, \mathbb{Z})\right) \neq 0$.

(ii) There are parallel exact sequences (see Section 2) that enable us to "translate" cohomological discussions in number theory into topological context word by word. Our Section 4 will be an example. For example, we will consider in Proposition 16 the Herbrand quotient $Q=\left(\# \widehat{H}^{0}\left(G, Z_{2}(N, \mathbb{Z})\right)\right) /\left(\# \widehat{H}^{1}\left(G, Z_{2}(N, \mathbb{Z})\right)\right)$ as an invariant of branched covers.

(2) Since Hilbert's Satz 90 is a very basic fact, we can expect various applications of this, such as analogues of Kummer theory, for example. An analogue of Hilbert's Satz 94 is also proved in [Mo2, Chapter 1].

(3) Lemma 9 and Theorems 10 and 11 hold for any dimension $n>1$; that is, we have $\widehat{H}^{1}\left(G, Z_{n-2}(N, \mathbb{Z})\right) \cong B_{n-1}(N, \mathbb{Z})^{G} / h^{!} B_{n-1}(M, \mathbb{Z})$ and $\widehat{H}^{1}\left(G, C_{n-2}(N, \mathbb{Z})\right)=0$ for covers of $n$-manifolds branched over $(n-2)$ submanifolds.

\section{$\S 4$. Proofs of main theorems}

In this section we prove our main theorems in Section 3. We will sometimes omit the coefficients $\mathbb{Z}$ to make the notation brief in the following.

Proof of Theorem 6. First, let us paraphrase the conclusion. By Hurewicz's theorem, we have $H_{1}(M, \mathbb{Z}) \cong \pi_{1}(M) / D\left(\pi_{1}(M)\right)$ for any manifold $M$, where $D(G):=[G, G]$ denotes the commutator group of a group $G$. Note also that we have $h_{*}\left(D\left(\pi_{1}(N)\right)\right) \triangleleft D\left(\pi_{1}(M)\right)$. Then we see that the following conditions are equivalent:

$$
\begin{aligned}
h_{*}: & H_{1}(N, \mathbb{Z}) \rightarrow H_{1}(M, \mathbb{Z}) \quad \text { is surjective } \\
& \Longleftrightarrow h_{*}: \pi_{1}(N) / D\left(\pi_{1}(N)\right) \rightarrow \pi_{1}(M) / D\left(\pi_{1}(M)\right) \quad \text { is surjective } \\
& \Longleftrightarrow \pi_{1}(M) \quad \text { is generated by } h_{*}\left(\pi_{1}(N)\right) \text { and } D\left(\pi_{1}(M)\right) .
\end{aligned}
$$

Now we prove the contraposition of the theorem. Suppose that $\pi_{1}(M)$ is not generated by $h_{*}\left(\pi_{1}(N)\right)$ and $D\left(\pi_{1}(M)\right)$, that is, that $\pi_{1}(M) \gtreqless h_{*}\left(\pi_{1}(N)\right)$. $D\left(\pi_{1}(M)\right) \triangleright D\left(\pi_{1}(M)\right)$. Then by Galois theory for covers, we have a nontrivial subcover $g: S \rightarrow M$ of the maximal (unbranched) abelian cover $M_{\mathrm{ab}} \rightarrow M$ which satisfies $\pi_{1}(S)=h_{*}\left(\pi_{1}(N)\right) \cdot D\left(\pi_{1}(M)\right)$, where the last term is the group generated by the elements of $h_{*}\left(\pi_{1}(N)\right)$ and $D\left(\pi_{1}(M)\right)$. 
Since $g: S \rightarrow M$ is a cover and $h: N \rightarrow M$ is a continuous map with $h_{*}\left(\pi_{1}(N)\right) \triangleleft \pi_{1}(S)$, we have a lift of $h$, a continuous map $f: N \rightarrow S$ with $h=g \circ f$, by general theory of cover (which is proved by using lifts of homotopies):
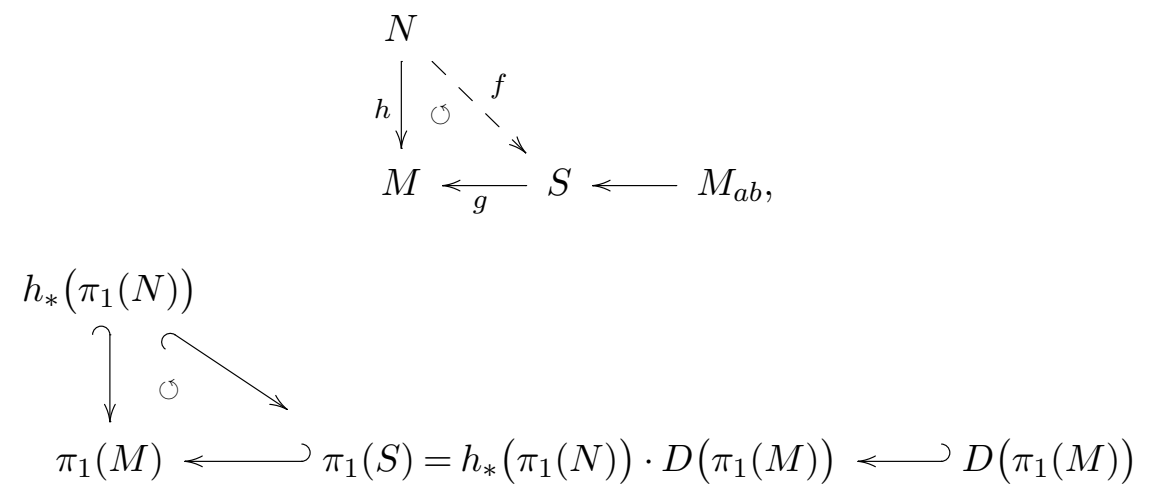

Thus, we obtain a nontrivial abelian subcover $g$ of $h$, and the contraposition is proved.

We give here a proof of Theorem 7 which is parallel to [W, proof of Theorem 2], a group-theoretic one. We can observe what is not parallel in this proof. We may have another proof by taking contraposition and by using the Wang sequence and the fact that $p \nmid \# H_{1}(M, \mathbb{Z}) \Longleftrightarrow H_{1}\left(M, \mathbb{F}_{p}\right)=0$.

Proof of Theorem 7. By Hurewicz's theorem and Galois theory, we see that $p \mid \# H_{1}(M)$ if and only if $M$ has an unbranched abelian cover of degree $p$.

When $h$ is a nontrivial unbranched cover, $\operatorname{Gal}(N / M)$ is a $p$-group, and it has a normal subgroup $G_{1}$ with index $p$, so we obtain a subcover $N / G_{1} \rightarrow M$, which is an unbranched cover of $M$ of degree $p$.

If $h$ is branched at a knot $K$, we denote by $g: H \rightarrow N$ the maximal $p$ abelian (unbranched) cover of $N$, we put $L:=\bigsqcup\left\{L_{i}\right\}=h^{-1}(K)$, and we put $J:=g^{-1}(L)=\bigsqcup\left\{J_{i j}\right\}$, where $g\left(J_{i j}\right)=L_{i}$.

We claim that the branched cover $f:=h \circ g: H \rightarrow M$ is Galois ...( $)$. (For number field extensions, this follows immediately from the maximality of $H$.) We check this later.

Now let $G$ be the Galois group of the branched cover $f: H \rightarrow M$, and let $I_{i j}<G$ be the inertia groups of knots $J_{i j}$ (see Section 2 for inertia groups). 
By the assumption that $p \mid \# H_{1}(N)$, the cover $H \rightarrow N$ is not trivial, and since $g: H \rightarrow N$ is unbranched, we have $\# I_{i j} \operatorname{deg}(h)<\operatorname{deg}(f)$, and hence $I_{i j} \supsetneqq G$.

Because $G$ is p-group, there is a normal subgroup $G_{1}$ of $G$ with index $p$ which includes one of inertia groups, say, $I_{11}$. But at this moment, since $f: H \rightarrow M$ is Galois, all the inertia groups are conjugate of $I_{11}$ in $G$, and all $I_{i j}$ are included in $G_{1}$. Therefore, $H / G_{1} \rightarrow M$ is an unbranched cover with degree $p$ :

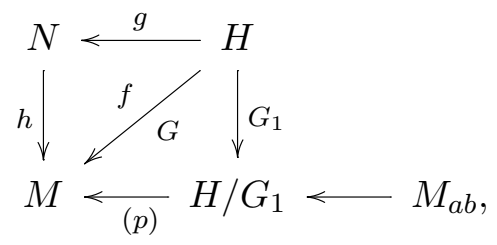

$$
\begin{array}{cccc}
\pi_{1}(N-L) & \triangleright & \pi_{1}(H-J) & \quad \\
\triangle & \nabla & & \\
& & & \\
\pi_{1}(M-K) & & &
\end{array}
$$

Proof of claim ( ). Let $X=M-K, Y=N-L, Z=H-J$ be the link complement spaces. Notice that $\pi_{1}(Z)$ is the pullback of the unique Syllow $p$-group $S_{p}$ of $H_{1}(N, \mathbb{Z})$ by the map $\pi_{1}(Y) \rightarrow \pi_{1}(N) \rightarrow \pi_{1}(N)^{a b} \cong H_{1}(N, \mathbb{Z})$.

Since $h: N \rightarrow M$ is Galois, $\pi_{1}(Y) \triangleleft \pi_{1}(X)$ is a normal subgroup, and we have an action of $\pi_{1}(X)$ on $\pi_{1}(Y)$ by conjugation: $\pi_{1}(X) \curvearrowright \pi_{1}(Y)$. Then, since $S_{p}$ is stable under the induced action of $\pi_{1}(X)$ on $H_{1}(N, \mathbb{Z})$, the group $\pi_{1}(Z)$, which is the pullback of $S_{p}$ in $\pi_{1}(Y)$, is also stable under the action $\pi_{1}(X) \curvearrowright \pi_{1}(Y)$. Therefore, $\pi_{1}(Z)$ is a normal subgroup of $\pi_{1}(X)$, and hence $f: H \rightarrow M$ is Galois.

Proof of Corollary 8. We prove the result when $m$ and $p$ are coprime, from which the other cases follow:

$$
M_{m p^{\nu}} \longrightarrow M_{m} \longrightarrow S^{3}
$$

Since the cover $M_{m p^{\nu}} \rightarrow M_{m}$ has no nontrivial unbranched subcover, Theorem 6 implies that $\# H_{1}\left(M_{m}\right) \mid \# H_{1}\left(M_{m p^{\nu}}\right)$, and hence that $p \mid$ $\# H_{1}\left(M_{m}\right) \Longrightarrow p \mid \# H_{1}\left(M_{m p^{\nu}}\right)$. 
On the other hand, since this is a cover of degree $p^{\nu}$ and its branching set is a knot $K$, Theorem 7 implies that $p\left|\# H_{1}\left(M_{m p^{\nu}}\right) \Longrightarrow p\right| \# H_{1}\left(M_{m}\right)$.

REMARK. In Corollary 3, only one prime $(p)$ is ramified in $\mathbb{Q}\left(\zeta_{p^{\nu}}\right) / \mathbb{Q}$, and its extension degree is $(p-1) p^{\nu-1}$. If we put $m=p-1$ in Corollary 8 , we get the strict analogue of Corollary 3.

Proof of Lemma 9 (Analogue of Hilbert's Satz 90). Since $h$ is Galois and $N$ is a $G$-complex with its branching set being 1-dimensional subcomplex, $C_{2}(N, \mathbb{Z})$ is a $\mathbb{Z}[G]$-free module. The result is immediate from this, because $\widehat{H}^{q}(G, F)=0$ for any $q \in \mathbb{Z}$, finite group $G$, and $G$-free module $F$ (see [B, Chapter 6]).

REMARK. Note that since multiplicative group of number field $\ell^{*}$ is not $\mathbb{Z}[G]$-free, Hilbert's Satz 90 for number field extensions is more nontrivial. It is proved by using Dedekind's lemma, or linear independence of automorphisms, which needs both addition and multiplication. We cannot prove the two lemmas in parallel ways.

Proof of Theorem 10. We omit the coefficients $\mathbb{Z}$ in the following. Note that $H^{1}(G)=,\widehat{H}^{1}(G$,$) .$

We consider the following short exact sequence of $\mathbb{Z}[G]$-module:

$$
0 \rightarrow Z_{2}(N) \rightarrow C_{2}(N) \stackrel{\partial}{\rightarrow} B_{1}(N) \rightarrow 0
$$

By taking Galois cohomology, we obtain a long exact sequence

$$
0 \rightarrow Z_{2}(N)^{G} \rightarrow C_{2}(N)^{G} \stackrel{\partial}{\rightarrow} B_{1}(N)^{G} \rightarrow H^{1}\left(G, Z_{2}(N)\right) \rightarrow H^{1}\left(G, C_{2}(N)=0,\right.
$$

where the last equality is by Lemma 9 .

Now the transfer map $h^{!}: C_{2}(M) \stackrel{\cong}{\rightrightarrows} C_{2}(N)^{G}$ and the map $(1 / n) h_{*}$ : $C_{2}(N)^{G \stackrel{\cong}{\longrightarrow}} C_{2}(M)$ induce the isomorphism

$$
B_{1}(M)=\partial\left(C_{2}(M)\right) \underset{h^{!}}{\stackrel{\cong}{\longrightarrow}} \partial\left(C_{2}(N)^{G}\right),
$$

and hence we have an exact sequence

$$
0 \rightarrow B_{1}(M) \stackrel{h^{!}}{\rightarrow} B_{1}(N)^{G} \rightarrow H^{1}\left(G, Z_{2}(N)\right) \rightarrow 0 .
$$

Proof of Theorem 11. We prove here that the Tate cohomology $\widehat{H}^{r}(G$, $\left.Z_{2}(N)\right)$ is independent of cellular decompositions of $N$, where $h: N \rightarrow M$ is 
a branched Galois cover branched over a link $L$ with Galois group $G$ and of degree $n=\# G$. We take one $G$-CW-structure on $N$ which has $h^{-1}(L)$ as a subcomplex. We denote the singular chains of $N$ by $C_{*}^{\text {sing }}(N)$, and so on.

The inclusion from cellular chains into singular chains leads to a commutative diagram of $G$-chain complexes with exact rows and columns:

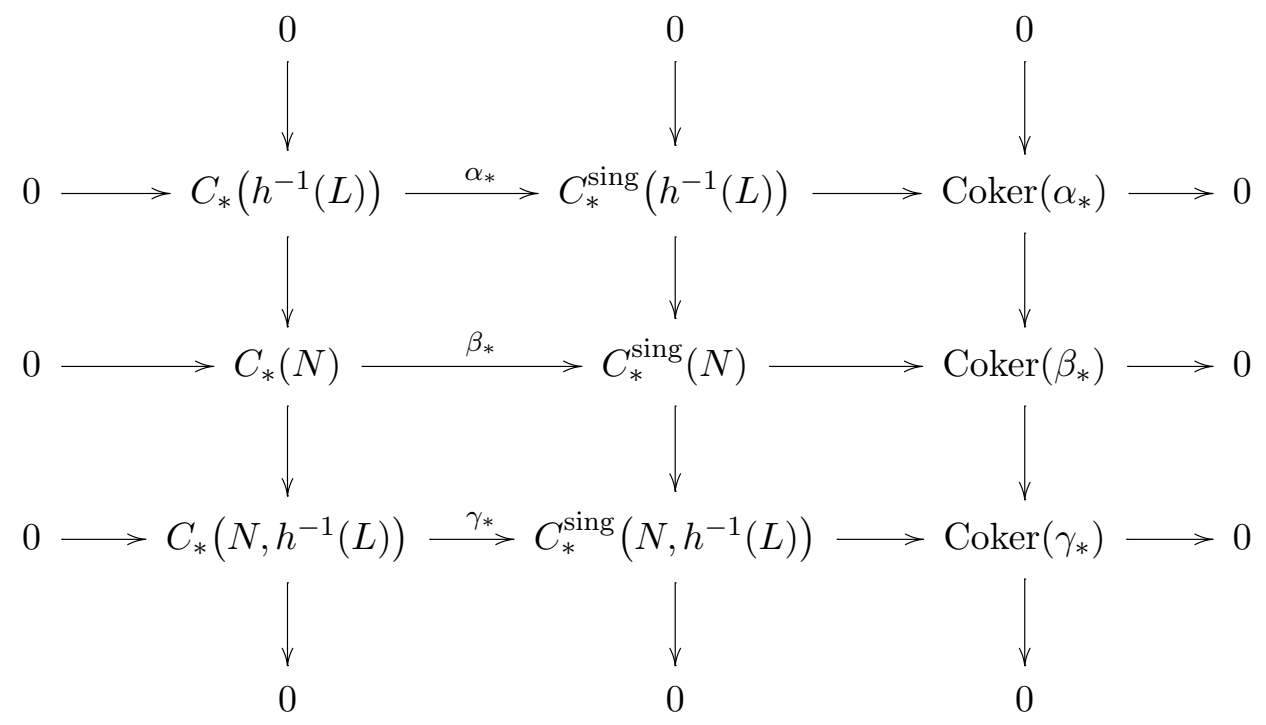

Since the inclusions $\alpha_{*}, \beta_{*}, \gamma_{*}$ are chain homotopy equivalences, the cokernels are acyclic complexes. Since $G$ acts freely away from $h^{-1}(L)$, the complexes in the third row are free $G$-modules. By diagram chasing (use $B_{q}\left(\operatorname{Coker}\left(\alpha_{*}\right)\right)=Z_{q}\left(\operatorname{Coker}\left(\alpha_{*}\right)\right)$ by acyclicity), we obtain exact sequences:

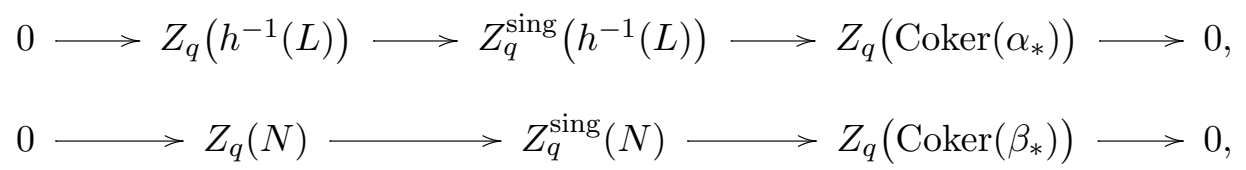

and so on. We also have an exact column $0 \longrightarrow Z_{q}\left(\operatorname{Coker}\left(\alpha_{*}\right)\right) \longrightarrow Z_{q}$ $\left(\operatorname{Coker}\left(\beta_{*}\right)\right) \longrightarrow Z_{q}\left(\operatorname{Coker}\left(\gamma_{*}\right)\right) \longrightarrow 0$. Since $h^{-1}(L)$ is 1-dimensional, 
$Z_{q}\left(h^{-1}(L)\right)=0$ for all $q>1$. Hence, we have following exact diagram:

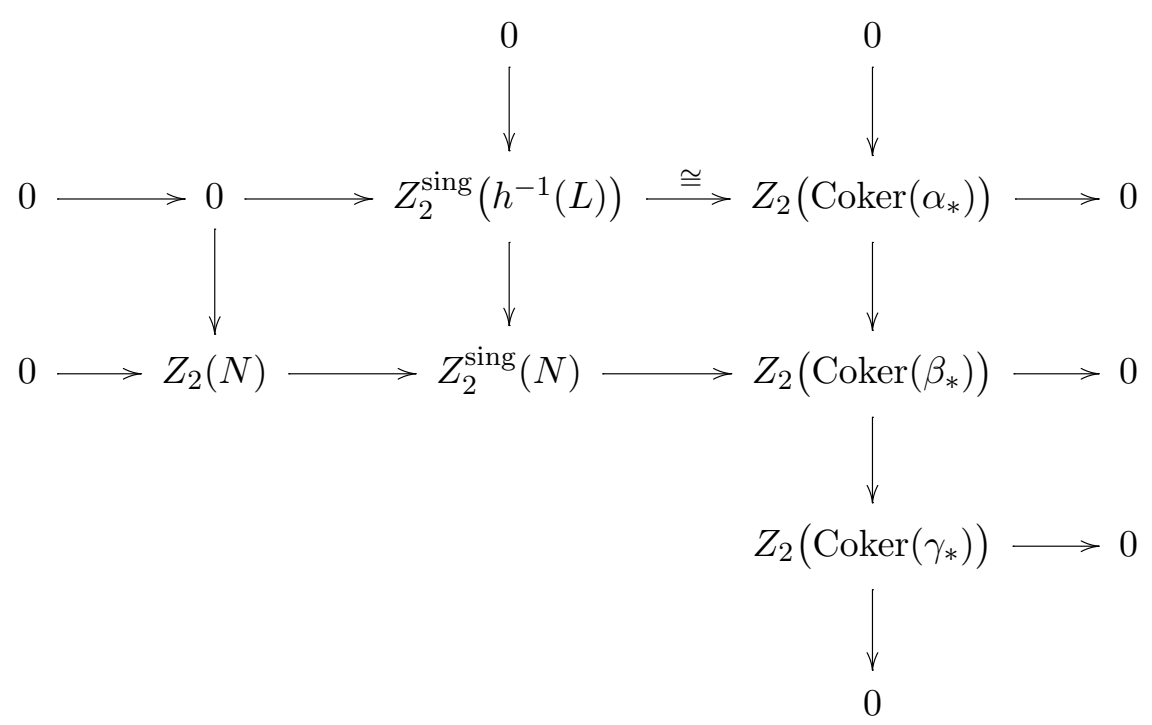

Hence, $0 \longrightarrow Z_{2}(N) \longrightarrow Z_{2}^{\text {sing }}(N) / Z_{2}^{\text {sing }}\left(h^{-1}(L)\right) \longrightarrow Z_{2}\left(\operatorname{Coker}\left(\gamma_{*}\right)\right) \longrightarrow 0$ is exact by the nine lemma, and $0 \longrightarrow \widehat{H}^{r}\left(G, Z_{2}(N)\right) \longrightarrow \widehat{H}^{r}\left(G, Z_{2}^{\text {sing }}(N)\right) \longrightarrow$ $\widehat{H}^{r}\left(G, Z_{2}\left(\operatorname{Coker}\left(\gamma_{*}\right)\right) \longrightarrow \widehat{H}^{r+1}\left(G, Z_{2}(N)\right) \longrightarrow 0\right.$ is exact by the snake lemma. Since $Z_{2}\left(\operatorname{Coker}\left(\gamma_{*}\right)\right)$ is $G$-free and $G$ is finite, $\widehat{H}^{r}\left(G, Z_{2}\left(\operatorname{Coker}\left(\gamma_{*}\right)\right)\right)=$ 0 for all $r \in \mathbb{Z}$, and hence $\widehat{H}^{r}\left(G, Z_{2}(N)\right) \stackrel{\cong}{\longrightarrow} \widehat{H}^{r}\left(G, Z_{2}^{\text {sing }}(N)\right)$ for all $r \in \mathbb{Z}$. The last group is independent of the cellular decomposition. (This proof holds for all $q>1$.)

\section{$\S 5$. Application to genus theory}

In this section, we give some genus-theory-type theorems obtained as applications of our Theorem 10. First, we recall the number field case. Here are some applications of Theorem 5 , which give balances between ideal class groups and ramification indices.

Lemma 12 ([Y, Lemma 1]). Let $\ell / k$ be a finite Galois extension of number fields with Galois group $G$. Then

$$
\# I(\ell)^{G} / P(\ell)^{G}=\# C l(k) \frac{\prod e}{\# \widehat{H}^{1}\left(G, \mathcal{O}_{k}^{*}\right)},
$$

where $\prod e$ is the product of the ramification indices of all the finite primes at $\ell / k$. 
Note that in the proof of Lemma 12 we use Theorem $5, \widehat{H}^{1}\left(G, \mathcal{O}_{\ell}^{*}\right) \cong$ $P(\ell)^{G} / P(k)$, and the fact that $\prod e=\left[I(\ell)^{G}: I(k)\right]$.

Proposition 13 ([Y, Lemma 6]). Let $\ell / k$ be a cyclic extension with Galois group $G$. Then, for the $G$-invariant subgroup of ideal class group $C l(\ell)^{G}$,

$$
\# C l(\ell)^{G}=\frac{\# \widehat{H}^{0}\left(G, \mathcal{O}_{\ell}^{*}\right) \cdot \prod e \cdot \# C l(k)}{\# \widehat{H}^{1}\left(G, \mathcal{O}_{\ell}^{*}\right) \cdot\left[\mathcal{O}_{k}^{*}:(\eta)\right]} .
$$

REMARK. If $\ell / k$ is abelian, $\# C l(\ell)^{G}$ is equal to what is called the relative genus number of $\ell / k$.

Proposition 14 ([Y, Lemma 3]). When a group $G$ is acting on $A$, $Q(A)=\# \widehat{H}^{0}(G, A) / \# \widehat{H}^{1}(G, A)$ is called the Herbrand quotient. For a cyclic extension $l / k$ with Galois group $G$, we have

$$
Q\left(\mathcal{O}_{\ell}^{*}\right):=\frac{\widehat{H}^{0}\left(G, \mathcal{O}_{\ell}^{*}\right)}{\widehat{H}^{1}\left(G, \mathcal{O}_{\ell}^{*}\right)}=\frac{\prod e_{\infty}}{n},
$$

where $\prod e_{\infty}$ is the product of the branching indices at the infinite primes.

Note. In order to see the analogy closely, we sketch the proof of Proposition 13. Precisely, we have

$$
\begin{aligned}
\# C l(\ell)^{G}= & {\left[\bigcup C l(\ell)^{G}: P(\ell) I(\ell)^{G}\right] \times\left[P(\ell) I(\ell)^{G}: P(\ell) I(k)\right] } \\
& \times[P(\ell) I(k): P(\ell)] \\
= & \frac{\# \widehat{H}^{0}\left(G, \mathcal{O}_{\ell}^{*}\right)}{\left[\mathcal{O}_{k}^{*}:(\eta)\right]} \times \frac{\prod e \cdot h_{0}}{\# \widehat{H}^{1}\left(G, \mathcal{O}_{\ell}^{*}\right)} \times \frac{\# C l(k)}{h_{0}}
\end{aligned}
$$

where $\widehat{H}^{0}\left(G, \mathcal{O}_{\ell}^{*}\right)=\mathcal{O}_{k}^{*} / N\left(\mathcal{O}_{\ell}^{*}\right),(\eta):=\mathcal{O}_{k}^{\times} \cap N(\ell)$, and $h_{0}=\# \operatorname{Ker}(T r:$ $C l(k) \rightarrow C l(\ell))$. Furthermore,

(i) $\left[\bigcup C l(\ell)^{G}: P(\ell) I(\ell)^{G}\right]=\left[(\eta): N\left(\mathcal{O}_{\ell}^{*}\right)\right]\left(=\# \widehat{H}^{0}\left(G, \mathcal{O}_{\ell}^{*}\right) /\left[\mathcal{O}_{k}^{*}:(\eta)\right]\right)$ is proved by the isomorphism $\bigcup C l(\ell)^{G} / P(\ell) I(\ell)^{G} \cong(\eta) / N\left(\mathcal{O}_{\ell}^{*}\right)$,

(ii) $[P(\ell) I(k): P(\ell)]=\# C l(k) / h_{0}$ is proved by applying the homomorphism theorem for the transfer map, and

(iii) $\left[P(\ell) I(\ell)^{G}: P(\ell) I(k)\right]=\prod e \cdot h_{0} / \# \widehat{H}^{1}\left(G, \mathcal{O}_{\ell}^{*}\right)$ follows from the fact that $P(\ell) I(\ell)^{G} / P(\ell) I(k) \cong I(\ell)^{G} / P(\ell)^{G}$, (ii), and Lemma 12. 
Next, we consider the 3-manifold analogues of the propositions above in this section, which are related to genus theory for 3-manifolds (see [Mo1], [Mo3], [Mo4]), as applications of our Theorem 10. They can be seen as more precise versions of theorems in [Mo1]. Note that we omit coefficients $\mathbb{Z}$ in the following.

Lemma 15 (Analogue of Lemma 12). Let $h: N \rightarrow M$ be a finite Galois cover over a rational homology 3-sphere $M$, of degree $n$ with Galois group $G$. Then

$$
\# Z_{1}(N)^{G} / B_{1}(N)^{G}=\frac{\# H_{1}(M) \cdot \prod e}{\# \widehat{H}^{1}\left(G, Z_{2}(N)\right)},
$$

where $\prod e$ is the product of the branching indices of all the branching knots of $h$.

Proof of Lemma 15. By our Theorem 10, $\widehat{H}^{1}\left(G, Z_{2}(N)\right) \cong B_{1}(N)^{G} /$ $h^{!} B_{1}(M)$, and by the fact that $\prod e=\left[Z(N)^{G}: h^{!} Z(M)\right]$, we obtain the following:

$$
\begin{aligned}
\# Z_{1}(N)^{G} / B_{1}^{G}= & {\left[Z_{1}(N)^{G}: B_{1}(N)^{G}\right] } \\
= & {\left[Z_{1}(N)^{G}: h^{!} Z_{1}(M)\right] } \\
& \cdot\left[h^{!} Z_{1}(M): h^{!} B_{1}(M)\right] /\left[B_{1}(N)^{G}: h^{!} B_{1}(M)\right] \\
= & \prod e \cdot \# H_{1}(M) / \# \widehat{H}^{1}\left(G, Z_{2}(N)\right) .
\end{aligned}
$$

Proposition 16 (Analogue of Proposition 13). Let $h: N \rightarrow M$ be a cyclic cover over a rational homology sphere $M$, with Galois group $G=\langle\sigma|$ $\left.\sigma^{n}=1_{G}\right\rangle$. Then, for the $G$-invariant subgroup of the first homology group $H_{1}(N, \mathbb{Z})^{G}$, we have

$$
\# H_{1}(N)^{G}=\frac{\# \widehat{H}^{0}\left(G, Z_{2}(N)\right)}{\# \widehat{H}^{1}\left(G, Z_{2}(N)\right)} \cdot \prod e \cdot \# H_{1}(M) .
$$

In particular, the Herbrand quotient $Q\left(Z_{2}(N)\right)=\left(\# \widehat{H}^{0}\left(G, Z_{2}(N)\right)\right) /$ $\left(\# \widehat{H}^{1}\left(G, Z_{2}(N)\right)\right)$ is an invariant of the cover.

REMARK. If $h: N \rightarrow M$ is abelian, the relative genus cover $N^{*} \rightarrow N$ is defined to be the maximal cover of $N$ which is an abelian cover over $M$ and is unbranched over $N$, and $g_{N / M}:=\operatorname{deg}\left(N^{*} \rightarrow N\right)$ is called the relative genus number with respect to $h: N \rightarrow M$. 
When $h: N \rightarrow M$ is a cyclic cover over an integral homology sphere $M$ (i.e., $H_{1}(M, \mathbb{Z})=0$ ), we have the following equalities proved by Morishita [Mo1]:

$$
\# H_{1}(N)^{G}=\left[H_{1}(N):(1-\sigma) H_{1}(N)\right]=\left[H_{1}(N): H_{1}\left(N^{*}\right)\right]=g_{N / M}=\frac{\prod e}{n} .
$$

Comparing with our equality in Proposition 16, we obtain the Herbrand quotient of $G$ acting on $Z_{2}(N)$, as follows.

Proposition 17. Suppose that $M$ is an integral homology sphere in the assumptions of Proposition 16; then

$$
Q\left(Z_{2}(N)\right)=\frac{\# \widehat{H}^{0}\left(G, Z_{2}(N)\right)}{\# \widehat{H}^{1}\left(G, Z_{2}(N)\right)}=\frac{1}{n} .
$$

This is an analogue of the Herbrand quotient for unit groups of cyclic extension of number fields $\ell / k$ with $\prod e_{\infty}=1$ (i.e., without branching at any infinite prime).

REMARKS.

(1) The Herbrand quotient of $Z_{2}(N)$ for general $M$ is yet to be calculated, because it is difficult to apply the technique used in [Mo1] for general $M$. However, we have following.

(i) For any branched Galois cover $h: N \rightarrow M$ with Galois group $G$, by definition we have

$$
\widehat{H}^{0}\left(G, Z_{2}(N)\right)=Z_{2}(N)^{G} /\left(\sum_{\sigma \in G} \sigma\right) Z_{2}(N)
$$

(ii) If $G$ is cyclic, by group cohomology theory and our Lemma 9, we also have

$$
\begin{aligned}
B_{1}(N)^{G} / h^{!} B_{1}(M) & \cong \widehat{H}^{1}\left(G, Z_{2}(N)\right) \cong \widehat{H}^{-1}\left(G, Z_{2}(N)\right) \\
& =\operatorname{Ker}\left(h_{*}: Z_{2}(N) \rightarrow h^{!} Z_{2}(M) /(1-\sigma) Z_{2}(N)\right) .
\end{aligned}
$$

(2) We note that topological analogues of the infinite primes are known to be ends of noncompact 3-manifolds (see [D], [Mo3], [Mo4], [Ra]). Therefore, since $M$ is closed, the term corresponding to the infinite primes disappears in Proposition 17. 
Proof of Proposition 16. We have more precise equations:

$$
\begin{aligned}
\# H_{1}(N)^{G}= & {\left[\bigcup H_{1}(N)^{G}: B_{1}(N)+Z_{1}(N)^{G}\right] } \\
& \times\left[B_{1}(N)+Z_{1}(N)^{G}: B_{1}(N)+h^{!} Z_{1}(M)\right] \\
& \times\left[B_{1}(N)+h^{!} Z_{1}(M): B_{1}(N)\right] \\
= & \# \widehat{H}^{0}\left(G, Z_{2}(N)\right) \times \frac{\prod e \cdot h_{0}}{\# \widehat{H}^{1}\left(G, Z_{2}(N)\right)} \times \frac{\# H_{1}(M)}{h_{0}},
\end{aligned}
$$

and each term coincides (so, they are integers), where $\widehat{H}^{0}\left(G, Z_{2}\right)=Z_{2}(M) /$ $h_{*}\left(Z_{2}(N)\right)$ and $h_{0}=\# \operatorname{Ker}\left(h^{!}: H_{1}(M) \rightarrow H_{1}(N)\right)$. Indeed,

(i) $\bigcup H_{1}(N)^{G} / B_{1}(N)+Z_{1}(N)^{G} \cong Z_{2}(M) / h_{*}\left(Z_{2}(N)\right)$ proves the coincidence of the first terms,

(ii) $B_{1}(N)+h^{!} Z_{1}(M) / B_{1}(N)$ is the image of transfer map $h^{!}: H_{1}(M) \rightarrow$ $H_{1}(N)$ with $\# \operatorname{Ker}\left(h^{!}\right)=h_{0}$, and

(iii) $\left[B_{1}(N)+Z_{1}(N)^{G}: h^{!}\left(Z_{1}(M)\right)+B_{1}(N)\right]=\left(\prod e \cdot h_{0}\right) /\left(\# \widehat{H}^{1}\left(G, Z_{2}(N)\right)\right)$ since $B_{1}(N)+Z_{1}(N)^{G} / B_{1}(N) \cong Z_{1}(N)^{G} / B_{1}^{G}$ and by Lemma 15 and (ii).

Proof of (i). In order to prove the isomorphism $\bigcup\left(H_{1}(N)^{G}\right) / B_{1}(N)+$ $Z_{1}(N)^{G \stackrel{\cong}{\rightrightarrows}} Z_{2}(M) / h_{*}\left(Z_{2}(N)\right)$, we consider the following map:

$$
\begin{aligned}
\varphi: \bigcup H_{1}(N)^{G} & \rightarrow Z_{2}(M) / h_{*}\left(Z_{2}(N)\right) \\
c & \mapsto\left[h_{*}(s)\right],
\end{aligned}
$$

where $(1-\sigma) c=\partial s$. We will define the map, show it is surjective, and make its kernel explicit.

First, for $c \in Z_{1}(N)$, the following equivalence and equation show that there is such $s$ :

$$
\begin{aligned}
c \in \bigcup H_{1}(N)^{G} & \Longleftrightarrow[c] \in H_{1}(N)^{G} \\
& \Longleftrightarrow(1-\sigma) c \in B_{1}(N) \\
& \Longleftrightarrow \exists s \in C_{2}(N), \quad \text { such that }(1-\sigma) c=\partial s,
\end{aligned}
$$

$\partial h_{*}(s)=h_{*}(\partial s)=0$.

Suppose that $(1-\sigma) c=\partial s=\partial s^{\prime} ;$ then $\partial\left(s-s^{\prime}\right)=0, s-s^{\prime} \in Z_{2}(N)$, and hence

$$
h_{*}(s)-h_{*}\left(s^{\prime}\right)=h_{*}\left(s-s^{\prime}\right) \in h_{*}\left(Z_{2}(N)\right) .
$$

Hence, the map $\varphi$ exists and is well defined. 
Next, for any $s \in Z_{2}(M)$, there exists $\tilde{s} \in C_{2}(N)$ such that $h_{*}(\tilde{s})=s$. Since $h_{*}(\partial s)=\partial h_{*}(s)=0$ and $\operatorname{Ker}\left(h_{*}\right)=\operatorname{Im}(1-\sigma)$ on $B_{1}(N)$, we have $\partial s=(1-\sigma) c$ for some $c \in B_{1}(N)$. Hence, $\varphi$ is surjective.

Lastly, take $c \in \bigcup H_{1}(N)^{G}$, and put $\varphi(c)=\left[h_{*}(s)\right]$ with $(1-\sigma) c=\partial s ;$ then the following equivalence shows the results:

$$
\begin{aligned}
c \in \operatorname{Ker} \varphi & \Longleftrightarrow h_{*}(s) \in h_{*}\left(Z_{2}(N)\right) \\
& \Longleftrightarrow s \in Z_{2}(N)+\operatorname{Ker}\left(h_{*}\right)=Z_{2}(N)+(1-\sigma) C_{2}(N) \\
& \Longleftrightarrow(1-\sigma) c=\partial s \in(1-\sigma) \partial C_{2}(N)=(1-\sigma) B_{1}(N) \\
& \Longleftrightarrow c \in B_{1}(N)+Z_{1}(N)^{G} .
\end{aligned}
$$

Note that since $\varphi(c)=\left[h_{*}(s)\right]$ is well defined, it is sufficient to consider any $s \in C_{2}(N)$ with $(1-\sigma) c=\partial s$ above.

Acknowledgments. I am sincerely grateful to Masanori Morishita for his kind guidance into arithmetic topology and lots of encouragement all along the way. I thank Mikio Furuta for instructive discussions and accurate suggestions. I also thank Jonathan Hillman for helpful advice and especially for the main idea behind the proof of Theorem 11, for which one of the referees also provided a necessary modification. In addition, I would like to thank T. Shimizu, K. Tokimoto, T. Mihara, K. Yahiro, and all my colleagues for enjoyable daily discussions.

\section{REFERENCES}

[B] K. S. Brown, Cohomology of Groups, Grad. Texts in Math. 87, Springer, New York, 1982. MR 0672956.

[D] C. Deninger, "A note on arithmetic topology and dynamical systems" in Algebraic Number Theory and Algebraic Geometry, Contemp. Math. 300, Amer. Math. Soc., Providence, 2002, 99-114. MR 1936368. DOI 10.1090/conm/300/05144.

[F] P. Furtwängler, Über die Klassenzahlen der Kreisteilungskörper, J. Reine Angew. Math. 140 (1911), 29-32.

[H] A. Hatcher, Algebraic Topology, Cambridge University Press, Cambridge, 2002. MR 1867354.

[HMM] J. Hillman, D. Matei, and M. Morishita, "Pro- $p$ link groups and $p$-homology groups" in Primes and Knots (Baltimore, 2003), Contemp. Math. 416, Amer. Math. Soc., Providence, 2006, 121-136. MR 2276139. DOI 10.1090/conm/416/ 07890 .

[I1] K. Iwasawa, A note on the group of units of an algebraic number field, J. Math. Pures Appl. (9) 35 (1956), 189-192. MR 0076803.

[I2] - A note on class numbers of algebraic number fields, Abh. Math. Semin. Univ. Hambg. 20 (1956), 257-258. MR 0083013. 
[I3] - On $\Gamma$-extensions of algebraic number fields, Bull. Amer. Math. Soc. (N.S.) 65 (1959), 183-226. MR 0124316.

[KM] T. Kadokami and Y. Mizusawa, Iwasawa type formula for covers of a link in a rational homology sphere, J. Knot Theory Ramifications 17 (2008), 1199-1221. MR 2460171. DOI 10.1142/S0218216508006580.

[K] M. M. Kapranov, "Analogies between the Langlands correspondence and topological quantum field theory" in Functional Analysis on the Eve of the 21st Century, Vol. 1. (New Brunswick, N.J., 1993), Progr. Math. 131, Birkhäuser, Boston, 1995, 119-151. MR 1372994.

[Ma] B. Mazur, Remarks on the Alexander polynomial, 1963-1964, http://www.math. harvard.edu/ mazur/papers/alexander_polynomial.pdf (accessed 3 October 2013).

[Mo1] M. Morishita, A theory of genera for cyclic coverings of links, Proc. Japan Acad. Ser. A Math. Sci. 77 (2001), 115-118. MR 1857286.

[Mo2] - "On capitulation problem for 3-manifolds" in Galois Theory and Modular Forms (Tokyo, 2001), Dev. Math. 11, Kluwer, Boston, 2004, 305-313. MR 2059769. DOI 10.1007/978-1-4613-0249-0_15.

[Mo3] - Analogies between knots and primes, 3-manifolds and number rings, Sugaku Expositions 23 (2010), 1-30. MR 2605747.

[Mo4] - Knots and Primes: An Introduction to Arithmetic Topology, Universitext, Springer, London, 2012. MR 2905431. DOI 10.1007/978-1-4471-2158-9.

[Mn1] B. Morin, Utilisation d'une cohomologie étale équivariante en topologie arithmétique, Compos. Math. 144 (2008), 32-60. MR 2388555. DOI 10.1112/ S0010437X07003168.

[Mn2] - Sur le topos Weil-étale d'un corps de nombres, Ph.D. dissertation, Université Bordeaux 1, Talence, France, 2008.

[Ra] N. Ramachandran, A note on arithmetic topology, C. R. Math. Acad. Sci. Soc. R. Can. 23 (2001), 130-135. MR 1869056.

[Re] A. Reznikov, Embedded incompressible surfaces and homology of ramified coverings of three-manifolds, Selecta Math. (N.S.) 6 (2000), 1-39. MR 1771215. DOI 10.1007/s000290050001.

[Se] J.-P. Serre, Local Fields, Grad. Texts in Math. 67, Springer, New York, 1979. MR 0554237.

[Si] A. Sikora, Analogies between group actions on 3-manifolds and number fields, Comment. Math. Helv. 78 (2003), 832-844. MR 2016698. DOI 10.1007/ s00014-003-0781-x.

[W] L. C. Washington, Introduction to Cyclotomic Fields, Grad. Texts in Math. 83, Springer, New York, 1982. MR 0718674. DOI 10.1007/978-1-4684-0133-2.

[Y] H. Yokoi, On the class number of a relatively cyclic number field, Nagoya Math. J. 29 (1967), 31-44. MR 0207681.

Graduate School of Mathematics

Kyushu University

Fukuoka-city, Fukuoka, 819-0395

Japan

uekijun46@gmail.com 